\title{
Transforming Pseudo-Triangulations
}

\author{
Sergei Bespamyatnikh \\ Department of Computer Science, University of Texas at Dallas, \\ Box 830688, Richardson, TX 75083, USA \\ besp@utdallas.edu
}

\begin{abstract}
We study the problem of transforming pseudo-triangulations in the plane. We show that a pseudo-triangulation with $n$ vertices can be transformed into another one using $O(n \log n)$ flips only. This improves the previous bound $O\left(n^{2}\right)$ [3]. We present an algorithm for computing a transformation between two pseudo-triangulations in $O((f+n) \log n)$ time where $f$ is the number of flips.
\end{abstract}

\section{Introduction}

Pseudo-triangles and pseudo-triangulations have received much attention recently because of applications in visibility [8,9], ray shooting [8], collision detection [7], and rigid motion $[4,10]$.

A pseudo-triangle is a simple polygon with exactly three convex vertices. For a set $S$ of $n$ points in the plane, a pseudo-triangulation $T$ is defined as partition of the convex hull of $S$ into interior disjoint pseudo-triangulations whose vertices are points of $S$ (each point of $S$ is a vertex of $T$ and vice versa). A minimum pseudo-triangulation is a pseudo-triangulation with the least number of edges among all pseudo-triangulations of $S$.

One of the operations on triangulations and pseudo-triangulations is the flip, see Fig. 1. Flips in pseudo-triangulations are used for enumeration all pseudotriangulations defined by a set of vertices $[1,3]$. These algorithms imply that a pseudo-triangulation with $n$ vertices can be transformed to any other using at most $O\left(n^{2}\right)$ flips.

In this paper we show that any two pseudo-triangulations with the same set of $n$ vertices can be transformed into each other by $O(n \log n)$ flips only. We present a simple algorithm for finding a sequence of flips based on our proof. The algorithm computes flips in $O((f+n) \log n)$ time where $f$ is the number of flips.

Interestingly, the flip distance between pseudo-triangulations is smaller than one between triangulations. Hurtado et al. [6] show that there are $2 n$ points in the plane and two triangulations that require more than $(n-1)^{2}$ flips to transform one into the other.

\section{Preliminaries}

Let $\operatorname{conv}(A)$ denote the convex hull of a set $A$ in the plane. We classify vertices of a polygon in the plane: convex vertices whose internal angle is less than $\pi$ 


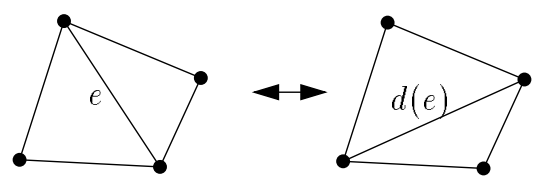

a)
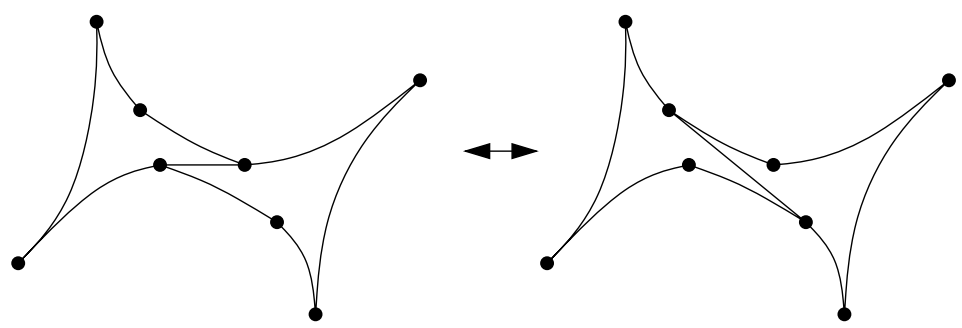

b)

Fig. 1. a) Flip of triangles. b) Flip of pseudo-triangles.

and reflex vertices whose internal angle is greater than $\pi$. A pseudo-triangle is a simple polygon with three convex vertices. A sequence of pseudo-triangle edges between two convex vertices is called a side of the pseudo-triangle.

We assume that $S$ is a set of $n$ points in the plane in general position (no three collinear points). Let $T$ be a pseudo-triangulation and $e$ be an edge of $T$ whose flip introduces an edge $e^{\prime}$. The edge $e^{\prime}$ is called a dual edge of E, see Fig. $1 \mathrm{~b})$.

An edge $e$ of a pseudo-triangle $\Delta$ is called cross edge for a vertex $a$ if $e$ is on the side $b c$ and a point of $e$ is visible from $a$. Note that $\Delta$ can have many cross edges for $a$.

Lemma 1. Let abc be a pseudo-triangle of a pseudo-triangulation $T$ and let $e$ be a cross edge for $a$. Let $\Delta$ be a pseudo-triangle with vertex a obtained by flipping e. The dual edge of $e$ is not a cross edge of a in $\Delta$.

Proof. First we note that there can be two pseudo-triangles incident to $a$ after flipping $e$, see, for example, Fig. 1 a). In this case the dual edge of $e$ (which is the common edge of two new pseudo-triangles) is incident to $a$ and is a cross edge in either pseudo-triangle.

In general, let $b c d$ be the pseudo-triangle that contains the edge $e$. The union of pseudo-triangles $a b c$ and $b c d$ is a pseudo-quadrangle, a simple polygon with exactly four convex vertices. The dual edge of $e$ is a part of geodesic path $a d$ in the pseudo-quadrangle $a b c d$, see, for example, Fig. $1 \mathrm{~b}$ ). The lemma follows. 


\section{Canonical Pseudo-Triangulations}

In this Section we introduce canonical pseudo-triangulations and show how a pseudo-triangulation can be transformed into a canonical one.

Definition 1 Let $S$ be a set of $n$ points in the plane and let $p$ be a vertex of the convex hull of $S$. Let $\sigma(p)=\left(p_{1}, p_{2}, \ldots, p_{n}\right)$ be a sorted sequence of points such that

(i) $p_{1}=p$, and

(ii) the points $p_{2}, \ldots, p_{n}$ are sorted in clockwise order around $p_{1}$, and

(iii) $p_{1} p_{2}$ and $p_{1} p_{n}$ are the edges of the convex hull, see Fig. 2.

$A$ canonical pseudo-triangulation with center $p$, denoted by $\mathcal{T}(p, S)$, is defined recursively as follows. If $n=3$ (if $n=1$ or $n=2 \mathcal{T}(p, S)$ has no pseudotriangles) then $\mathcal{T}(p, S)$ consists of one triangle $p_{1} p_{2} p_{3}$. Suppose that $n \geq 4$. Let $m=\lceil n / 2\rceil$ and $S_{1}=\left\{p_{1}, p_{2}, \ldots, p_{m}\right\}$ and $S_{2}=\left\{p_{1}, p_{m+1}, p_{m+2}, \ldots, p_{n}\right\}$. $\mathcal{T}(p, S)$ contains the pseudo-triangles of $\mathcal{T}\left(p, S_{1}\right)$ and $\mathcal{T}\left(p, S_{2}\right)$ and a pseudo-

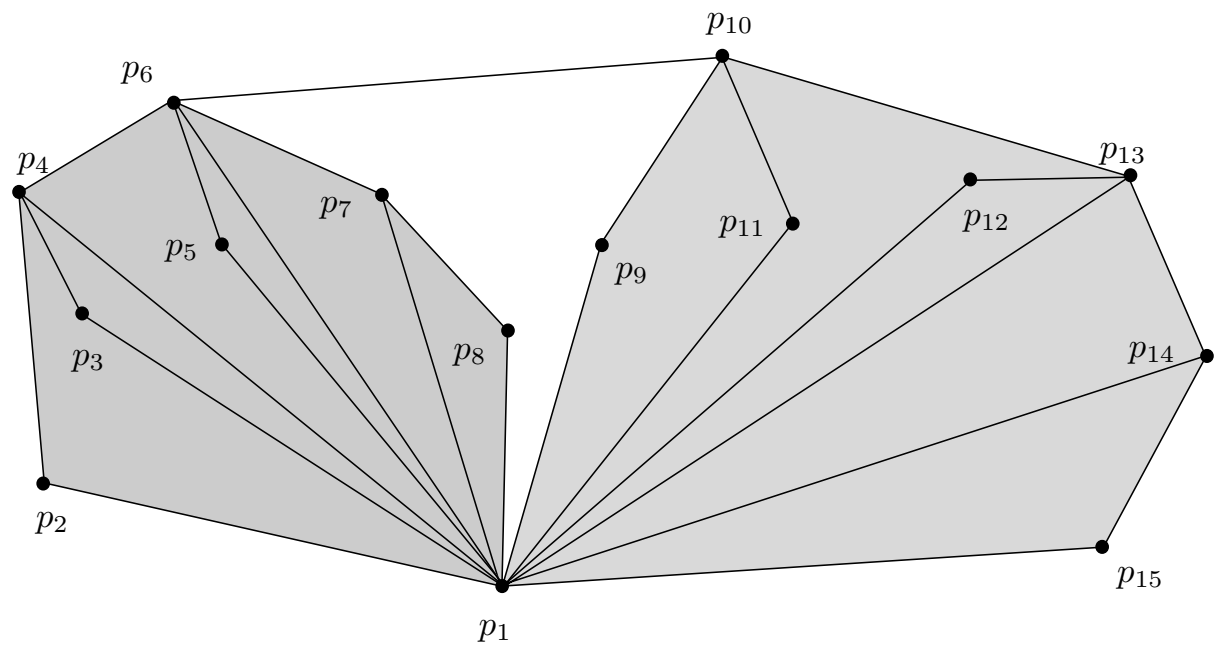

Fig. 2. Canonical pseudo-triangulation with center $p_{1}$.

triangle that remains if $\operatorname{conv}\left(S_{1}\right)$ and $\operatorname{conv}\left(S_{2}\right)$ is removed from $\operatorname{conv}(S)$, see Fig. 2.

Theorem 2. Let $S$ be a set of $n$ points in the plane in general position and let $p$ be a point of $S$. Let $T$ be a pseudo-triangulation of $S$. The canonical pseudotriangulation with center $p$ can be obtained from $T$ using $O(n \log n)$ flips. 
Proof. We apply flips to $T$ and construct the canonical pseudo-triangulation $\mathcal{T}(p, S)$ as follows. If $S$ contains three points then $S$ then they define the unique triangle and $T$ contains it. Suppose that $S$ has at least four points. We consider the sets $S_{1}$ and $S_{2}$ from Definition 1. Let $P=\operatorname{conv}(S)$ and $P_{i}=\operatorname{conv}\left(S_{i}\right)$ where $i=1,2$. Let $\Delta$ be the pseudo-triangle $\Delta=P \backslash P_{1} \cup P_{2}$, see Fig. 3. Let $p_{i_{1}}$ and $p_{i_{2}}$ be two convex vertices of $\Delta$ where $i_{1}<i_{2}$.

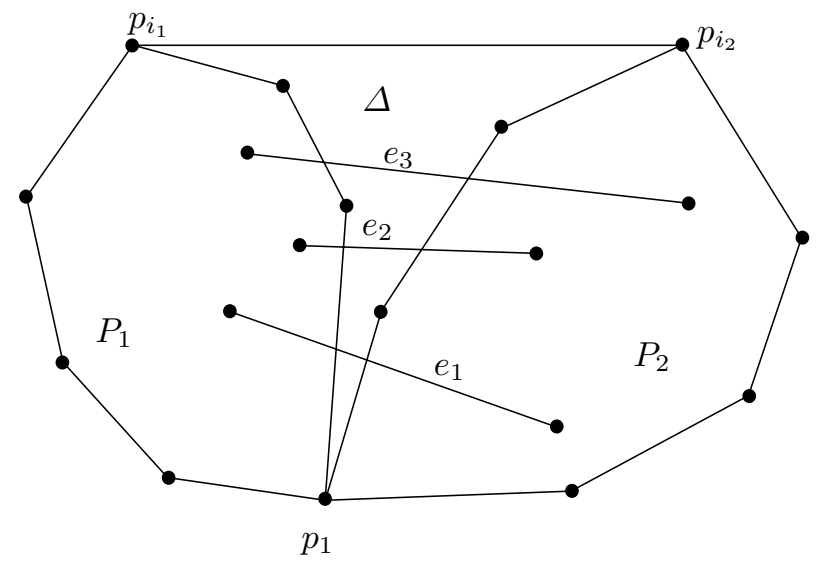

Fig. 3. Edges $e_{1}, e_{2}, \ldots, e_{l}$.

If an edge $e$ of the pseudo-triangulation $T$ has both endpoints in $S_{1}\left(\right.$ or $S_{2}$ ) then $e$ does not intersect $\Delta$. Thus, if an edge, say $e=\left(p_{i}, p_{j}\right), i<j$, intersects $\Delta$ then $p_{i}$ is in $S_{1}$ and $p_{j}$ is in $S_{2}$. Therefore $e$ crosses the pseudo-triangle $\Delta$. Let $E=\left\{e_{1}, e_{2}, \ldots, e_{l}\right\}$ be a set of the edges of $T$ intersecting $\Delta$ where the edges of $E$ are in the order they intersect the side $p_{1} p_{i_{1}}$, see Fig. 3. Let $s$ be a segment in $\Delta$ that connect $p_{1}$ and a point of the segment $p_{i_{1}} p_{i_{2}}$.

First we show that, if the set $E$ is empty then the pseudo-triangle $\Delta$ is present in $T$. Suppose that $E=\emptyset$. The segment $s$ lies in a pseudo-triangle of $T$ since none of edges of $T$ intersects $s$. Let $\Delta^{\prime}$ denote this pseudo-triangle. Clearly, $p_{i_{1}} p_{i_{2}}$ is an edge of $\Delta^{\prime}$. Let $C_{j}$ where $j=1,2$ be the side of $\Delta$ between $p_{1}$ and $p_{i_{j}}$. It remains to show that the pseudo-triangle $\Delta^{\prime}$ contains all the edges of $C_{1}$ and $C_{2}$. Suppose that an edge $e=\left(p_{j_{1}} p_{j_{2}}\right)$ of $C_{1}$ is not the edge of $\Delta^{\prime}$. The edge $e$ split $\Delta^{\prime}$ into two polygons, see Fig. 4 . Let $Q$ be the polygon that does not contain $s . Q$ has a convex vertex different from $p_{j_{1}}$ and $p_{j_{2}}$ (vertex $p_{k}$ in Fig. 4 ). The vertices $p_{1}, p_{i_{1}}$ and $p_{i_{2}}$ are convex vertices of $\Delta^{\prime}$ too. Contradiction.

By Lemma 1 the dual edge of $e_{1}$ does not intersect the segment $s$. We flip edges $e_{1}, e_{2}, \ldots, e_{l}$ in this order. Let $T^{\prime}$ be the pseudo-triangulation after $l$ flips. By the previous argument ( $E$ is empty) $T^{\prime}$ contains the pseudo-triangle $\Delta$. Note that $p_{1}$ is included in both $S_{1}$ and $S_{2}$. We apply flips recursively to the sets $S_{1}$ and $S_{2}$. Clearly, this yields the canonical pseudo-triangulation $\mathcal{T}(p, S)$. 


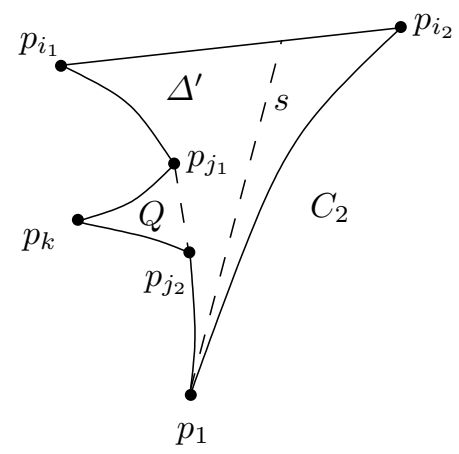

Fig. 4. Edge $p_{j_{1}} p_{j_{2}}$ must be in $\Delta^{\prime}$.

It remains to show that the total number of flips is $O(n \log n)$. The number of flips needed to generate the pseudo-triangle $\Delta$ is $l . l=O(n)$ since the total number of edges of $T$ is linear in terms of the number of vertices (a better bound $l \leq 2 n-3$ follows from the fact that $T$ is a pseudo-triangulation). Let $F(k)$ be the number of flips required by our procedure applied to a pseudo-triangulation with $k$ vertices. Then

$$
F(n)=O(n)+F(\lceil n / 2\rceil)+F(\lfloor n / 2\rfloor+1), F(1)=F(2)=F(3)=0 .
$$

$F(n)=O(n \log n)$ is the solution of the recurrence. The theorem follows.

\section{Algorithm}

Theorem 3. Let $S$ be a set of $n$ points in the plane in general position and let $T$ and $T^{\prime \prime}$ be two pseudo-triangulations defined on $S$. A sequence of $f=O(n \log n)$ flips transforming $T$ into $T^{\prime}$ can be found in $O((f+n) \log n)$ time.

Proof. Let $T$ and $T^{\prime \prime}$ be two pseudo-triangulations defined on the same set of vertices $S$. We show how to compute a sequence of flips that transforms $T$ into $T^{\prime}$. Let $p$ be a point on the convex hull of $S$. We apply flips according to Theorem 2. The algorithm computes a sequence of flips to transform $T$ into the canonical pseudo-triangulation $\mathcal{T}(p, S)$ and a sequence of flips to transform $T^{\prime}$ into the canonical pseudo-triangulation $\mathcal{T}(p, S)$. Combining these two sequences we obtain the transformation of $T$ into $T^{\prime}$.

The main computational difficulty is the finding of a dual edge. A simple linear time algorithm leads to $O(f n)$ total running time which can be superquadratic. We use an approach from [1] for maintaining a pseudo-triangulation. It is based on a data structure for storing a planar subdivision [5]. Applied to pseudo-triangulations the data structure has the following components:

- faces. The faces are pseudo-triangles. With each face we store pointers to its sides. 
- sides of pseudo-triangles. Each side is a sequence of edges that form a convex chain. We store the chain edges in counterclockwise order. We associate a binary tree with every chain. With each side we store a pointer to its pseudotriangle.

- edges. An edge is pair of vertices and we store pointers to these vertices. An edge is incident to either one pseudo-triangle (if its edge of $\operatorname{conv}(S)$ ) or two pseudo-triangles (otherwise). We keep two pointers for these sides.

- vertices. Each vertex is stored one time. With each vertex we store its incident edges.

It is shown [1] that a dual edge can found in $O(\log n)$ time and the data structure can be maintained in $O(\log n)$ time per flip. Therefore the total time for flipping edges and maintaining the data structure is $O(f \log n)$.

It remains to show how to compute the flipping edges. The algorithm for finding flips is recursive we show it for one recursive call. First we compute the segment $s$ that divides vertices of $S$ into two subsets of almost equal size. $s$ has the median slope and the median can be found in linear time [2].

We compute the edges $e_{1}, e_{2}, \ldots, e_{l}$ crossing the segment $s$ by checking every edge of $T$. In order to find the order of the edges we compute incident sides of pseudo-triangles and the pseudo-triangles traversed by $s$. This allows us to find the order in linear time.

Let $t(n)$ be the total time for computing flipping edges. Then $t(n)=O(n)+$ $2 t(n / 2)$ and the theorem follows.

\section{Conclusion}

We presented an algorithm that transforms a pseudo-triangulation to another one. The number of flips produced by the algorithm if $f=O(n \log n)$ where $n$ is the number of vertices. The running time of the algorithm is $O((f+n) \log n)$. An interesting open question is to close the gap between the trivial lower bound $f=\Omega(n)$ and the upper bound $f=O(n \log n)$ for the smallest number of flips $f$.

\section{References}

1. S. Bespamyatnikh. Enumerating pseudo-triangulations in the plane. In Proc. 14th Canad. Conf. Comput. Geom., pp. 162-166, 2002.

2. M. Blum, R. W. Floyd, V. Pratt, R. L. Rivest, and R. E. Tarjan. Time bounds for selection. J. Comput. Syst. Sci., 7:448-461, 1973.

3. H. Brönnimann, L. Kettner, M. Pocchiola, and J. Snoeyink. Counting and enumerating pseudo-triangulations with the greedy flip algorithm. In Fall Workshop on Comput. Geometry, 2001.

4. R. Connelly, E. D. Demaine, and G. Rote. Straightening polygonal arcs and convexifying polygonal cycles. In Proc. 41th Annu. Sympos. on Found. of Computer Science, pp. 432-442, 2000. 
5. M. de Berg, M. van Kreveld, M. Overmars, and O. Schwarzkopf. Computational Geometry: Algorithms and Applications. Springer-Verlag, Berlin, Germany, 2nd edition, 2000.

6. F. Hurtado, M. Noy, and J. Urrutia. Flipping edges in triangulations. Discrete Comput. Geom., 22(3):333-346, 1999.

7. D. Kirkpatrick, J. Snoeyink, and B. Speckmann. Kinetic collision detection for simple polygons. In Proc. 16th Annu. ACM Sympos. Comput. Geom., pp. 322330, 2000.

8. M. Pocchiola and G. Vegter. Pseudo-triangulations: Theory and applications. In Proc. 12th Annu. ACM Sympos. Comput. Geometry, pp. 291-300, 1996.

9. M. Pocchiola and G. Vegter. Topologically sweeping visibility complexes via pseudo-triangulations. Discrete Comput. Geom., 16(4):419-453, 1996.

10. I. Streinu. A combinatorial approach to planar non-colliding robot arm motion planning. In Proc. 41st Annu. IEEE Sympos. Found. Comput. Sci., pp. 443-453, 2000. 